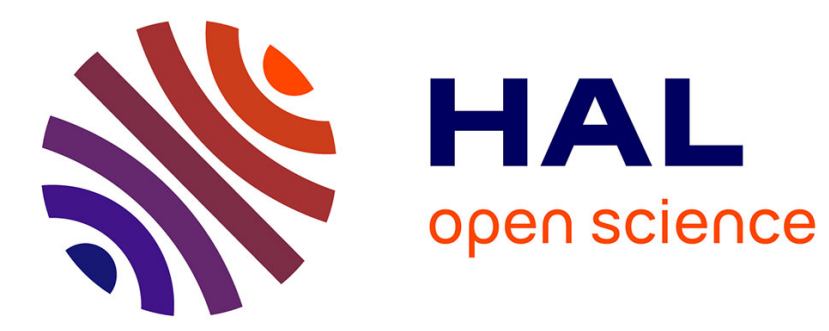

\title{
Bayesian curved lane estimation for autonomous driving
}

\author{
Mohamed Fakhfakh, Lotfi Chaâri, Nizar Fakhfakh
}

\section{To cite this version:}

Mohamed Fakhfakh, Lotfi Chaâri, Nizar Fakhfakh. Bayesian curved lane estimation for autonomous driving. Journal of Ambient Intelligence and Humanized Computing, 2020, 11, pp.4133-4143. 10.1007/s12652-020-01688-7 . hal-02950806

\section{HAL Id: hal-02950806 https://hal.science/hal-02950806}

Submitted on 28 Sep 2020

HAL is a multi-disciplinary open access archive for the deposit and dissemination of scientific research documents, whether they are published or not. The documents may come from teaching and research institutions in France or abroad, or from public or private research centers.
L'archive ouverte pluridisciplinaire HAL, est destinée au dépôt et à la diffusion de documents scientifiques de niveau recherche, publiés ou non, émanant des établissements d'enseignement et de recherche français ou étrangers, des laboratoires publics ou privés. 


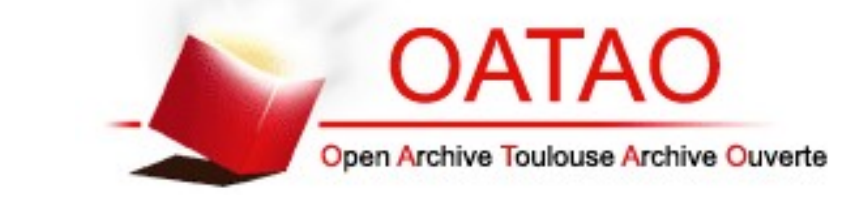

Open Archive Toulouse Archive Ouverte

OATAO is an open access repository that collects the work of Toulouse researchers and makes it freely available over the web where possible

This is an author's version published in:

http://oatao.univ-toulouse.fr/26409

\section{Official URL}

https://doi.org/10.1007/s12652-020-01688-7

To cite this version: Fakhfakh, Mohamed and Chaari, Lotfi and Fakhfakh, Nizar Bayesian curved lane estimation for autonomous driving. (2020) Journal of Ambient Intelligence and Humanized Computing. ISSN 1868-5137

Any correspondence concerning this service should be sent to the repository administrator: tech-oatao@listes-diff.inp-toulouse.fr 


\title{
Bayesian curved lane estimation for autonomous driving
}

\author{
Mohamed Fakhfakh ${ }^{1}$, Lotfi Chaari ${ }^{2}$, \\ and Nizar Fakhfakh ${ }^{3}$
}

\begin{abstract}
Several pieces of research during the last decade in intelligent perception are focused on the development of algorithms allowing vehicles to move efficiently in complex environments. Most of existing approaches suffer from either processing time which do not meet real-time requirements, or inefficient in real complex environment, which also doesn't meet the full availability constraint of such a critical function. To improve the existing solutions, an algorithm based on curved lane detection by using a Bayesian framework for the estimation of multi-hyperbola parameters is proposed to detect curved lane under challenging conditions. The general idea is to divide a captured image into several parts. The trajectory is modeled by a hyperbola over each part, whose parameters are estimated using the proposed hierarchical Bayesian model. Compared to the existing works in the state of the art, experimental results prove that our approach is more efficient and more precise in road marking detection.
\end{abstract}

Keywords Autonomous driving · embedded camera · road marking · multi-hyperbola $\cdot$ Bayesian framework

\footnotetext{
${ }^{1}$ University of Sfax, MIRACL laboratory, Tunisia

E-mail: mohamed.fakhfakh.research@gmail.com

${ }^{2}$ University of Toulouse, IRIT-ENSEEIHT, France

E-mail: lotfi.chaari@toulouse-inp.fr

${ }^{3}$ NAVYA, France

E-mail: nizar.fakhfakh@navya.tech
} 


\section{Introduction}

Autonomous driving is one of the main concerns which allows a step by step evolution of Artificial Intelligence. Indeed, it is important to ensure the proper functioning of autonomous vehicle mechanisms by making appropriate and accurate decisions in real time by identifying and distinguishing the different objects of the road.

In this direction, several studies have been carried out on autonomous driving in order to improve road security. The methods of obtaining intelligent vehicles systems are solely based on artificial intelligence, by trying reproducing human intelligence, and more precisely Machine Learning / Deep leaning approaches. The main goal of research and development of smart vehicles is to reduce accident rates, as well as improve the efficiency of traffic use by detecting different dangerous situations.

As regards the data, the state of the art studies generally rely on embedded equipments on the autonomous vehicles such as cameras (mono Nguyen et al. (2015), stereo Song et al. (2018) or multi-camera Ieng et al. (2005)) or other sensors like Odometer Santana et al. (2013), IMU Anwary et al. (2018), Differential GPS Chengping et al. (2014) or Lidars Kim and Park (2017b). Embedded processors allow running algorithms in order to move successfully from a starting point to a predefined destination. The main difficulties to perform this task generally lie in the possible presence of other vehicles and barriers Zhou and Dong (2017) as well as the recognition of road signs Botekar and Mahalakshmi (2017). Some works focus on the detection and classification of the road signs by using approaches based on three-step algorithms: color segmentation, shape recognition, and a neural network Broggi et al. (2007), Kim (2019). Deep learning approaches are being recently studied by using spatial transformers and stochastic optimization methods Arcos-García et al. (2018). An interesting survey study is done in Saxena et al. (2015).

Among all of self-driving functions, the lateral positioning of autonomous cars remain one of the most important tasks which should be carefully addressed. The lateral positioning might be performed by using "Simultaneous Localization and Mapping" approaches which allows a global localization process. Such methods from some intrinsic limits related to the problem formulation : whatever the used sensors for SLAM approaches Bresson et al. (2017), Shenoy et al. (2019) (GPS, Lidar, Camera), the lateral positioning do not work if the environment presents some repetitive patterns or in case of a lack of data typically for rural or forestry environments. In order to resolve that limits, a more complete scheme needs to be studied combining global and local positioning. Most of local positioning approaches were proposed by using embedded cameras but most of existing ones are not applicable in real deployment context. A state of the art will be presented and detailed in the next Section. Lateral positioning is a part of a more general function which allows a car to be driven by itself. Authors in Che et al. (2019) have recently proposed an interesting work focuced on path planning by using improved Particle Swarm 
Optimization.

In that context, our research is to find a trade-off between processing time and the efficiency of road marking detection and lane estimation in complex and challenging environments. The main contribution we propose is twise : the effectiveness and the precision of our model to estimate the curvature of road marking in challenging environments, and the process to divide a given image into three regions (one region for straight lanes and two regions for curved ones). This process allows well initialization of model's parameters.

In this work, we are focusing on methods allowing vehicles to have precise lateral positioning. Specifically, we are interested in the detection and estimation of road marking. Due to the importance of the lane detection task, researchers carried out many studies for several years on lane detection. In general, vision-based lane detection can be categorized into two main classes: straight and curve lanes.

This paper is organized as follows. After an introduction covering the context of this research, Section 2 is dedicated to the state of the art. In Section 3 , an overview of the proposed method is presented including all steps for road marking detection using images from embedded cameras. Evaluation and experimental results are detailed in Section 4. Finally, conclusions and future works are drawn in Section 5.

\section{Related Work}

There has been a significant amount of research on vision-based road lane detection. Many methods for straight and curved lane detection have been proposed during the last decade.

\subsection{Straight lanes}

The most efficient technique for straight lanes detection and estimation with reduced memory constraints is the Hough Transform (HT) Hassanein et al. (2015); Lopez-Krahe and Pousset (1988); Mukhopadhyay and Chaudhuri (2015). This technique has become a standard tool in the field of artificial vision. This step is usually performed either on original images obtained with the projective model or after applying an inverse perspective transform $\mathrm{Wu}$ and Chen (2016). The performance of HT algorithms highly depends on the amount of data.

In Kultanen et al. (1990); Xu et al. (1990), the authors proposed a particular HT who is Randomized Hough Transform (RHT). The difference between HT and RHT is that HT maps every single pixel to the accumulator space, whereas RHT handles pairs of pixels that manage a subset. As another extension, the Probabilistic Hough Transform (PHT) Guo et al. (2008) solves the 
same problem with low computational cost. In Liu et al. (2012), the Progressive Probabilistic Hough Transform (PPHT) has been proposed to minimize the proportion of points that are used in the vote while maintaining false negative and false positive rates close to those obtained by the standard HT. Some further works on binary images like generalized Hough transform or generalized fuzzy Hough transform and some others can be found in Antolovic (2008); Chiu et al. (2012); Izadinia et al. (2009); Thomas (1992).

In most methods, HT has been combined with other methods such as line classification. In Cela et al. (2013), presented an algorithm to detect road lanes based on an unsupervised and adaptive classifier. Three steps are used to obtain the lanes. In the first one, the brightness is used in the input image to know the environmental conditions and to highlights color lanes. The unsupervised classifier and HT are applied in the second step in order to identify the right and left road lines. A Kalman filter is applied after that to estimate the vehicle position and track the lane. The work in Liu et al. (2018) combined the Inverse Perspective Mapping (IPM) and K-Means Clustering to find out the correct lanes, and a HT to detect the line in the image. In Parajuli et al. (2013), the authors introduced a method for lane detection when shadows and low illumination conditions occur. The first step consists of recognizing the points on the adjacent right and left lanes using local gradient descriptors. Then, a simple linear prediction model leads to the detection of lane marking points on each horizontal stripes.

Other approaches are based on color features and HT for the sake of efficiency. In Bottazzi et al. (2014), the authors introduced a new method for lane marking detection based on HSV (Hue, Value, Saturation) histograms. A dynamic region of interest (ROI) is determined using an earlier triangle model. The first step is to calculate the histogram of the whole image. The illumination changes are found out by determining the difference between two successive images. The lane markers are segmented from the ROI. Then, a tracking Bouguet (2001) is used to validate and detect the lanes. In Li et al. (2018), the authors proposed another method based on the connection of various feature extraction. The main goal is to use the HSV color space to extract the white features of the road. Then, preliminary edge feature detection is added in the preprocessing step. For detection step, both Hough and Canny operators are applied. Finally, a Kalman Filter is applied to finalize lines tracking.

When real-time constraints are imposed, the authors in Lin et al. (2010) proposed a new approach based by extending the edge linking algorithm. An ROI is first initialized and then pixels belonging to edges are found using the Sobel operator. A directional edge-gap closing algorithm is proposed to produce more complete edge-links that possibly belong to the lanes. The next step consists of detecting the lane markers color. The fitting of the straight line model is then performed using a HT. 


\subsection{Curved lanes}

For curved lane detection, others works used more complex models such as B-Splines, parabolas, and hyperbolas Jung and Kelber (2005); Khalifa et al. (2010); Timar and Alagoz (2010). Bezier curves Cimurs et al. (2017) and BSplines Li et al. (2017b) are considered as relevant methods for curved lines detection, and have been configured to overcome the disadvantages of curves interpolation Dagnino et al. (2015) that suffer from two main drawbacks: i) the curve is defined by a polynomial whereas it is often more interesting to have a parametric representation, and ii) high computational cost since the polynomial degree increases in proportion to the number of control points.

For the same model that uses the B-splines method, the authors in Faizal and Mansor (2009); Wang et al. (1999) proposed an algorithm based on the BSnake technique. It is able to recover a wide range of lanes, especially curves. B-Snake is an implementation of B-Splines, so it can form an arbitrary shape by a set of control points. The system aims at finding both sides of way markings. This is achieved by detecting the mid line inside the lane and then estimating the other parallel lines. The initial position of the B-Snake is decided using the CHEVP algorithm (Canny / Hough Estimation of Vanishing Points). Then, to determine the control points of the B-Snake model, a minimization step is essential. The main drawback of this method is that it is not applicable in a real-time context, in contrast to the method in Aly (2008) where a robust and real-time algorithm is proposed for detecting lanes in urban streets. The top view model of the road images is generated using the inverse perspective mapping to reduce the perspective effect. The algorithm was based on taking filtering with Gaussian kernels. A similar idea has been developed in Guo et al. (2015) where an improved RANSAC algorithm is proposed to estimate the lane model parameters.

Authors in Lee et al. (2019) proposed a method based on deep learning from surround view images for autonomous driving of a ground vehicle evaluated on various unfavorable conditions with high-curvature lanes. In Wang et al. (2008), the authors use a hyperbola model for marking detection. A nonlinear term is integrated into the model to manage the transitions between the straight and curved segments of the road. The model parameters are estimated using the vanishing point which corresponds to the asymptotes of the hyperbola. In Chen and Wang (2006); Wang and An (2010) the authors proposed an algorithm based on a mixture of the linear-hyperbolic model. The general idea is to divide the road marking into two parts: near and far region from the camera. The curve detection in these two regions is based on a linear-hyperbolic model. For the nearby region, the HT method is applied since lanes are assumed to be straight. However, for the distant regions, a hyperbolic model is used for the case where the lines are curved. The most important parameter to estimate by this model is the curvature degree, as shown the following equation of the hyperbolic model: 


$$
y=\frac{a}{x-h}+b(x-h)+v .
$$

In $(2), x, y$ are the coordinates in pixels of the road marking points, $a$ is the curvature parameter of the hyperbola, $b$ is the slope of straight lane marking, and $(h, v)$ is the coordinate vector of the vanishing point. An approach has been proposed in Tan et al. (2014), which uses a hyperbola model, with an improvement by proposing a "River Flow" Lim et al. (2012) to detect curved lanes in difficult conditions, including dashed line markings and vehicle occlusion. In order to determine the curvature coefficient, a new method called "Improved River Flow" Tan et al. (2015) was proposed to search the characteristic points in the far field that corresponds to the lane marking.

In Jang et al. (2014), another kind of approaches is proposed based on the geometrical model. The general idea of the method is to apply two main steps: the first one involves the extraction of the road characteristics, and the second step relies on a geometrical model for lane detection. the candidate lines are detected by applying a voting system to extract the one that better fits the lanes. In Chen and He (2012), the authors proposed a method to detect sharp curve lanes using the maximum likelihood principle.

The third class of methods is based on learning techniques for lane extraction. For example, in Fan et al. (2013), the authors introduced a lane detection algorithm based on an improved Boosting algorithm using a classification function in order to classify whether each point is the edge point of the lane line. The authors in Kim et al. (2017) used a new learning algorithm for Convolutional Neural Networks (CNN) using an extreme learning machine (ELM) for image enhancement and lane detection. In Kim and Lee (2014), the authors used CNN to take off the noise in images and then used a RANSAC algorithm to fit lanes. The work in Kim and Park (2017a) proposed a sequential end-to-end transfer learning method two-time transfer learning to estimate left and right lanes without any postprocessing. In Li et al. (2017a), the authors proposed a detection model based on Deep Learning. A multitask network structure based on Recurrent Neural Networks (RNN) and CNN was designed to detect the lane boundaries including those areas containing no marks, without any explicit prior knowledge or secondary modeling.

Based on the literature review above, we can conclude that most existing methods for straight and curved lane detection provide good performance only for images with high quality are available. Moreover, sharply curved lane detection is still a challenging issue. Parametric models using hyperbolic representations are the most efficient in terms of the quality of lanes detection and parameter estimation. In this study, we focus on these models to propose a novel curved lanes characterization and estimation. The next Section details the proposed method. 


\section{Proposed method}

We detail here the proposed method for curved lanes estimation. Fig. 1 illustrates the general workflow of the proposed method.

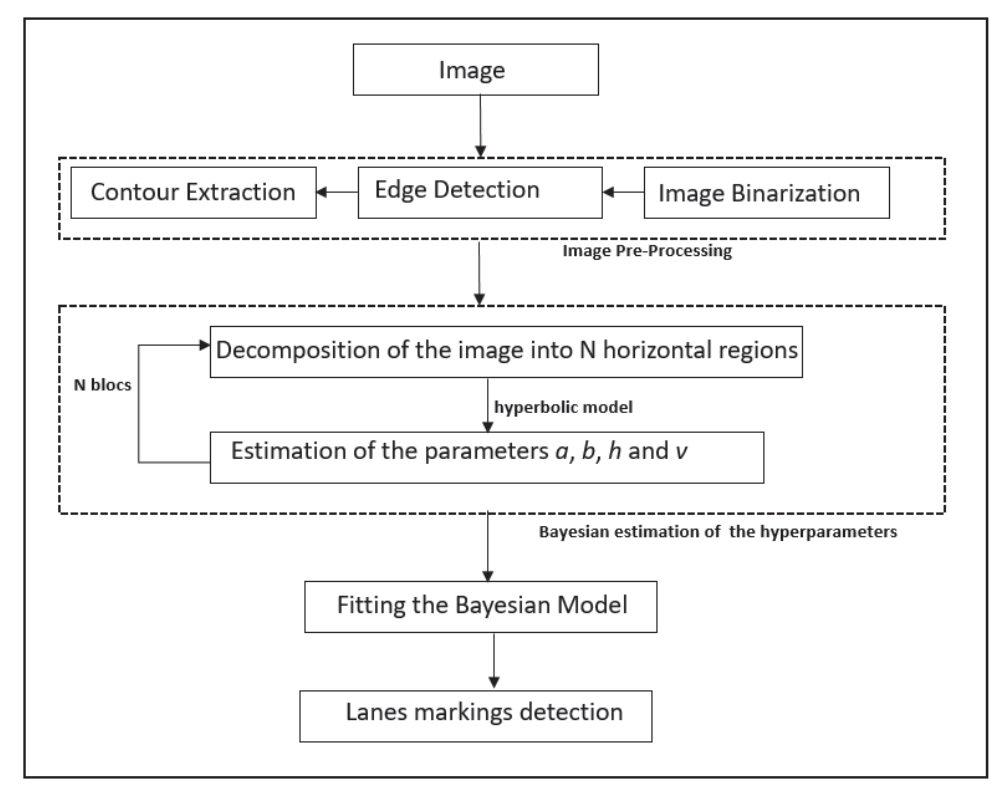

Fig. 1 General workflow of the of proposed method.

The input image goes through a preprocessing step in order to perform contour extraction like in Fakhfakh et al. (2018). The objective is then to characterize the extracted lanes by fitting them to the adopted analytical model. Specifically, the curved line is supposed to locally follow the hyperbolic model in (1). The image is therefore decomposed into $N$ horizontal regions as illustrated in Fig. 2, where a different hyperbola is associated with each region. The number of regions $(\mathrm{N})$ is empirically chosen. Each image is divided into three (3) different regions from the horizon line to the bottom of the image (near, medium, and far regions) and for each region the model is applied to estimate curvature parameters. This is motivated by the fact that according to the perspective geometry, the curvature is not constant along the image. A Bayesian model is proposed to allow estimating the hyperparameters of the $N$ hyperboles, and thus to accurately characterize the curved lane over the whole image. Indeed, Bayesian models are being more and more used in the signal and image literature due to their flexibility and high automatic level Andrieu (1998); Roumeliotis and Bekey (2000); Shiffrin et al. (2008). In this context, 
MCMC sampling is generally adopted to perform the inference Cowles and Carlin (1996); Merali and Barfoot (2013).

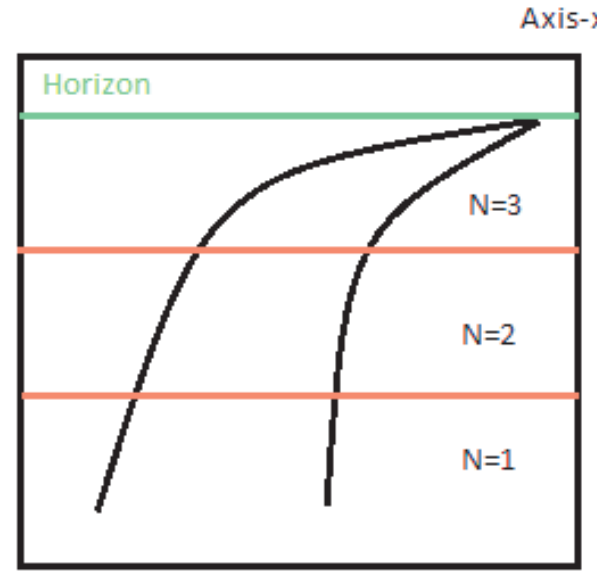

Axis-y

Fig. 2 The image is divided into $N$ horizontal blocks $(N=3)$.

\subsection{The adopted model}

According to the state of the art that we described above, we have noticed that the model proposed in Lim et al. (2012); Tan et al. (2014, 2015) based on hyperbola road model corresponds the most accurate road model than the others works (parabolic model, B-snake model, geometric model, etc.). This model allows finding solutions that meet the needs of autonomous vehicle users under complex environmental conditions. Adopting this analytical model, characterizing the curved lanes reduces to estimating the hyperparameters involved in the equation (1).

In this paper, we reformulate the curved lane characterization as an inverse problem. Specifically, a denoising problem is considered where the coordinate $y$ of the hyperbolic lane model in (1) is assumed to be an observation of the second coordinate $x$. Accounting for the horizontal decomposition of the image into $N$ regions, and for each pixel belonging to the lane, the following observation model is adopted

$$
y=\left[\sum_{i=0}^{N-1}\left(\frac{a_{i}}{x-h_{i}}+b_{i}\left(x-h_{i}\right)+v_{i}\right) 1_{\left[c_{i}, c_{i+1}\right]}(x)\right]+n
$$


where $n$ is a noise term assumed to be a Gaussian and $1_{[c i, c i+1]}$ is the indicator function which is equal to 1 on the interval $\left[c_{i}, c_{i+1}\right]$ and 0 otherwise. For the sake of representation, we adopt the following notations:

$-\mathbf{a}=\left\{a_{0}, \ldots, a_{N-1}\right\}$ is the vector grouping the parameters $a_{i}$;

$-\mathbf{b}=\left\{b_{0}, \ldots, b_{N-1}\right\}$ is the vector grouping the parameters $b_{i}$;

$-\mathbf{h}=\left\{h_{0}, \ldots, h_{N-1}\right\}$ is the vector grouping the parameters $h_{i}$;

$-\mathbf{v}=\left\{v_{0}, \ldots, v_{N-1}\right\}$ is the vector grouping the parameters $v_{i}$;

- $\mathbf{c}=\left\{c_{0}, \ldots, c_{N}\right\}$ is the vector grouping the parameters $c_{i}$.

The aim of the proposed method is therefor to estimate the hyperparameter vectors $\mathbf{a}, \mathbf{b}, \mathbf{h}, \mathbf{v}$ and $\mathbf{c}$, based on the observed coordinate vectors $\mathbf{y}=\left\{y_{1}, \ldots, y_{L}\right\}$ and $\mathbf{x}=\left\{x_{1}, \ldots, x_{L}\right\}$ where $L$ is the number of pixels in the lane to characterize.

\subsection{Hierarchical Bayesian model}

We propose to estimate the hyperparameter vector $\theta=\{\mathbf{a}, \mathbf{b}, \mathbf{c}, \mathbf{h}, \mathbf{v}\}$ using a Bayesian model. For doing so, the hyperparameter vectors $\mathbf{a}, \mathbf{b}, \mathbf{c}, \mathbf{h}$ and $\mathbf{v}$ are assumed to be realizations of random variables $A, B, C, H$ and $V$. In the following, we detail the adopted likelihood and prior models.

\subsubsection{Likelihood}

Based on the adopted observation model where the perturbation $n$ is assumed to be Gaussian with variance $\sigma_{n}^{2}$, the likelihood can be expressed as

$f(\mathbf{y} \mid \mathbf{x}, \mathbf{a}, \mathbf{b}, \mathbf{h}, \mathbf{v}, \mathbf{c}) \propto \prod_{l=1}^{L} \exp \left[\frac{-\left\|y_{l}-\sum_{i=0}^{N-1}\left(\frac{a_{i}}{x_{l}-h_{i}}+b\left(x_{l}-h_{i}\right)+v_{i}\right) 1_{\left[c_{i}, c_{i+1}\right]}\left(x_{l}\right)\right\|_{2}^{2}}{2 \sigma_{n}^{2}}\right]$.

\subsubsection{Hyperparameter priors}

Assuming the independence between the $N$ hyperboles, we associate here an inverse Gamma (IG) prior to each $a_{i}$ :

$$
\begin{aligned}
f\left(a_{i} \mid \alpha_{a}, \beta_{a}\right) & =\mathcal{I} \mathcal{G}\left(a_{i} \mid \alpha_{a}, \beta_{a}\right) \\
& \propto\left(a_{i}\right)^{-1-\alpha_{a}} \exp \left(-\frac{\beta_{a}}{a_{i}}\right) .
\end{aligned}
$$

Accounting for the $N$ Hyperboles, the joint prior writes 


$$
f\left(\mathbf{a} \mid \alpha_{a}, \beta_{a}\right) \propto \prod_{i=0}^{N-1} f\left(a_{i} \mid \alpha_{a}, \beta_{a}\right) .
$$

The shape and scale parameters $\left(\alpha_{a}\right.$ and $\left.\beta_{a}\right)$ can be either manually set or estimated by adding hyperpriors on them. It is worth noting that the IG prior is a common choice to model real-positive variables.

Since $\mathbf{b}, \mathbf{h}$ and $\mathbf{v}$ are also real-positive, the same strategy as for $\mathbf{a}$ has been used to define the requested priors: $f\left(\mathbf{b} \mid \alpha_{b}, \beta_{b}\right)=\prod_{i=0}^{N-1} \mathcal{I} \mathcal{G}\left(b_{i} \mid \alpha_{b}, \beta_{b}\right), f\left(\mathbf{h} \mid \alpha_{h}, \beta_{h}\right)=$ $\prod_{i=0}^{N-1} \mathcal{I} \mathcal{G}\left(h_{i} \mid \alpha_{h}, \beta_{h}\right), f\left(\mathbf{v} \mid \alpha_{v}, \beta_{v}\right)=\prod_{i=0}^{N-1} \mathcal{I} \mathcal{G}\left(v_{i} \mid \alpha_{v}, \beta_{v}\right)$.

As regards the hyperparameter $\mathbf{c}$, a uniform distribution on the interval $[0, M]$ is adopted for each $c_{i}$, where $M=\max \left\{x_{1}, \ldots, x_{L}\right\}$ :

$$
f(\mathbf{c})=\prod_{i=0}^{N} f\left(c_{i}\right)=\prod_{i=0}^{N} \mathcal{U}_{[0, M]}\left(c_{i}\right) .
$$

For the sake of simplicity, the noise variance $\sigma_{n}^{2}$ is supposed to be fixed to a small value in order to model a small perturbation level. However, this hyperparameter can also be estimated by fixing a suitable prior (such as the Jeffrey's one) to integrate it in the hierarchical model.

\subsection{Bayesian inference}

The Bayesian inference is built here to derive the estimators $\widehat{\mathbf{b}}, \widehat{\mathbf{h}}$ and $\widehat{\mathbf{v}}$ and $\widehat{\mathbf{c}}$.

\subsubsection{The proposed Gibbs sampler}

Following a maximum a posteriori (MAP) approach, the likelihood and the priors detailed in the previous section have to be combined in order to derive the posterior distribution and calculate the target estimators. Denoting by $\theta=$

$\{\mathbf{a}, \mathbf{b}, \mathbf{c}, \mathbf{h}, \mathbf{v}\}$ the target parameter vector and by $\Phi=\left\{\alpha_{a}, \beta_{a}, \alpha_{b}, \beta_{b}, \alpha_{h}, \beta_{h}, \alpha_{v}, \beta_{v}\right\}$, the joint posterior writes

$$
\begin{aligned}
& f(\theta \mid \mathbf{y}, \mathbf{x}, \Phi) \propto f(\mathbf{y} \mid \mathbf{x}, \mathbf{a}, \mathbf{b}, \mathbf{h}, \mathbf{v}, \mathbf{c}) f\left(\mathbf{a} \mid \alpha_{a}, \beta_{a}\right) f\left(\mathbf{b} \mid \alpha_{b}, \beta_{b}\right) f\left(\mathbf{h} \mid \alpha_{h}, \beta_{h}\right) f\left(\mathbf{v} \mid \alpha_{v}, \beta_{v}\right) f(c) \\
& \propto \prod_{l=1}^{L} \exp \left[\frac{-\| y_{l}-\left.\sum_{i=0}^{N-1}\left(\frac{a_{i}}{x_{l}-h_{i}}+b\left(x_{l}-h_{i}\right)+v_{i}\right) 1_{\left[c_{i}, c_{i+1}\right]}\left(x_{l}\right)\right|_{2} ^{2}}{2 \sigma_{n}^{2}}\right] \times \\
& \times\left(a_{i}\right)^{N\left(-1-\alpha_{a}\right)}\left(b_{i}\right)^{N\left(-1-\alpha_{b}\right)}\left(h_{i}\right)^{N\left(-1-\alpha_{h}\right)}\left(v_{i}\right)^{N\left(-1-\alpha_{v}\right)} \times \\
& \prod_{i=0}^{N-1} \exp \left(-\frac{\beta_{a}}{a_{i}}-\frac{\beta_{b}}{b_{i}}-\frac{\beta_{h}}{h_{i}}-\frac{\beta_{v}}{v_{i}}\right) \times \prod_{i=0}^{N} \mathcal{U}_{[0, M]}\left(c_{i}\right) .
\end{aligned}
$$


The objective here is to estimate the parameter and hyperparameter vectors $\theta$ and $\Phi$ based on the joint posterior in (7). However, it is clear that this posterior is difficult to handle. Deriving analytical estimators for $\theta$ and $\Phi$ is therefore a complicated task. For this reason, we resort to an MCMC sampling scheme, specifically a Gibbs sampler to numerically sample from the conditional distributions $f(\mathbf{a} \mid \mathbf{x}, \mathbf{y}, \Phi), f(\mathbf{b} \mid \mathbf{x}, \mathbf{y}, \Phi), f(\mathbf{h} \mid \mathbf{x}, \mathbf{y}, \Phi), f(\mathbf{v} \mid \mathbf{x}, \mathbf{y}, \Phi)$ and $f(\mathbf{c} \mid \mathbf{x}, \mathbf{y})$. The principle is to derive the conditional distribution for each unknown parameter ( in $\theta$ and $\Phi$ ) by integrating with respect to the other variables. These conditional distributions are then used to derive estimators, either by performing analytic calculations, or by resorting to numerical simulation techniques. As already stated, we resort here to numerical sampling using and MCMC scheme. The proposed Gibbs sampler is summarized in Algorithm 1.

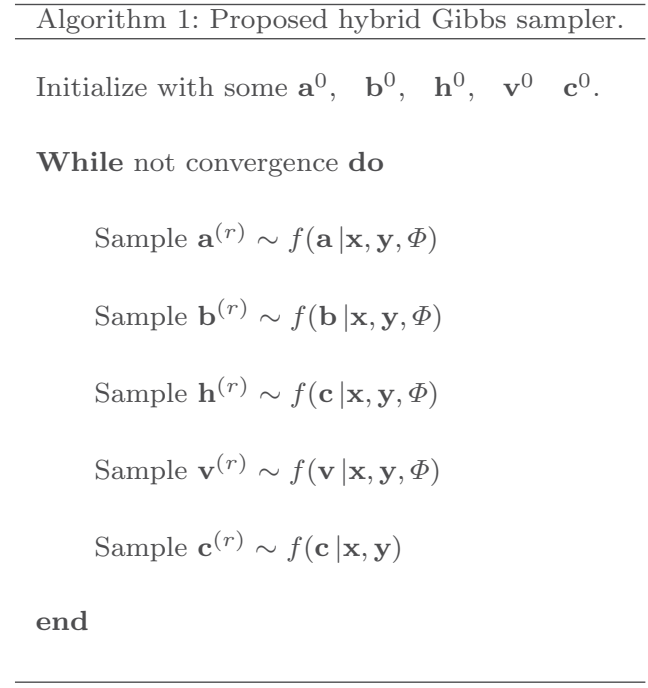

A burn-in period is essential so that the Gibbs sampled can reach convergence. The sampled chains $\left(\left(\mathbf{a}_{r}\right)_{1 \leq r \leq R},\left(\mathbf{b}_{r}\right)_{1 \leq r \leq R},\left(\mathbf{h}_{r}\right)_{1 \leq r \leq R},\left(\mathbf{v}_{r}\right)_{1 \leq r \leq R}\right.$ and $\left.\left(\mathbf{c}_{r}\right)_{1 \leq r \leq R}\right)$ are then used to derive estimators using for example the minimum mean square error (MMSE) estimator. The samples corresponding to the burn-in period are discarded.

In the following, the different conditional distributions are provided.

\subsubsection{Conditional distributions}

In order to derive the conditional distribution related to each parameter of the model, one has to integrate the joint posterior in (7) with respect to all the other parameter. As regards the hyperparameter $a_{i}$, calculations based on (7) 
lead to the following form

$$
\begin{aligned}
f\left(a_{i} \mid \mathbf{x}, \mathbf{y}, \Phi\right) & \propto \prod_{l=1}^{L} \exp \left[\frac{-\left\|y_{l}-\sum_{i=0}^{N-1}\left(\frac{a_{i}}{x_{l}-h_{i}}+b\left(x_{l}-h_{i}\right)+v_{i}\right) 1_{\left[c_{i}, c_{i+1}\right]}\left(x_{l}\right)\right\|_{2}^{2}}{2 \sigma_{n}^{2}}\right] \\
& \times\left(a_{i}\right)^{-1-\alpha_{a}} \exp \left(-\frac{\beta_{a}}{a_{i}}\right)
\end{aligned}
$$

By analysing the expression of this conditional distribution, it turns out that it is impossible to match it with one of the standard distributions (Gaussian, Gamma, Beta,...). A Metropolis-Hasting (MH) algorithm is used to sample according to this posterior since direct sampling is impossible to perform even if the obtained distribution belongs to the exponential family.

Due to the similarity of the used priors, similar calculations lead to conditional distributions of the same type for $f\left(b_{i} \mid \mathbf{x}, \mathbf{y}, \Phi\right), f\left(h_{i} \mid \mathbf{x}, \mathbf{y}, \Phi\right), f\left(v_{i} \mid \mathbf{x}, \mathbf{y}, \Phi\right)$ and $f\left(c_{i} \mid \mathbf{x}, \mathbf{y}, \Phi\right)$, and this by integrating (7) with respect to $b_{i}, h_{i}, v_{i}$ and $c_{i}$, respectively. Indeed, it is expected to have similar forms, the exponential structure is partly imposed by the adopted Gaussian likelihood. For the sake of conciseness, the full expressions of these conditionals are not detailed here. Simulation according to these conditionals is performed using an MH step.

\section{Experimental results}

In this Section, the proposed method for curved lane characterization is validated on both synthetic and real data in order to asses its performance.

\subsection{Simulated Data}

In the first experiment, the proposed algorithm was tested on synthetic data to validate its effectiveness. Each image is divided in three regions $(N=3)$ where in each region an hyperbola is estimated (except the first region from which a straight line is estimated). Fig. 3 illustrates the lane image with the simulated hyperboles assuming known parameters $\mathbf{a}, \mathbf{b}, \mathbf{c}, \mathbf{h}$, and $\mathbf{v}$.

Based on ground truth from which real road markings are available, the simulation was performed according to the observation model in (2). It is important to test different values of variance $\sigma_{n}^{2}$ in order to verify the accuracy of the model (processing time and estimated values of each hyperparameters) after convergence of the algorithm.

For doing so, we have chosen to test three differents variance values $\sigma_{n}^{2}=$ $30, \sigma_{n}^{2}=15$ and $\sigma_{n}^{2}=5$. This can allow us to measure the impact of the gaussian perturbation of the data on the algorithm performance, and this under 


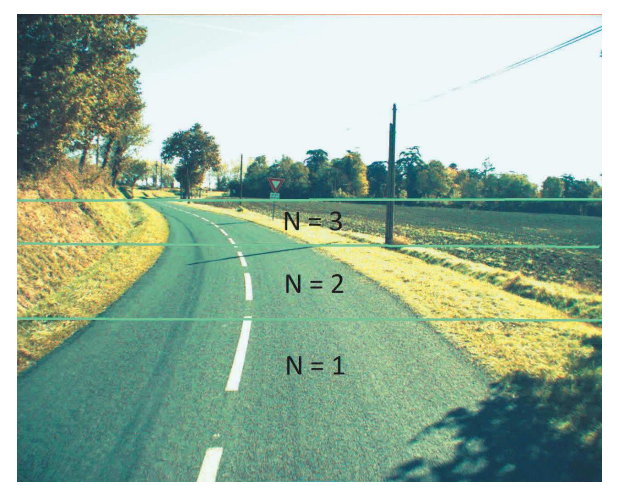

Fig. 3 The adopted ground truth for lane simulation with $N=3$ hyperboles.

more or less severe experimental conditions. The parameters of each hyperbola are then estimated using the proposed hybrid Gibbs sampler. 30.000 iterations were necessary to obtain the target estimators, among which 15.000 iterations were considered for the burn-in period to reach convergence.

Table 1 Hyperboles parameters for $\sigma_{n}^{2}=30$ : estimated and reference values. Average error is $3.64 \%$ and minimal error is $2.31 \%$

\begin{tabular}{|c|c|c|c|}
\hline Parameters & Ground truth & Estimated value & Error rate \\
\hline$a_{1}$ & 200 & 186 & $1.14 \%$ \\
\hline$b_{1}$ & 0.3 & 0.15 & $2.07 \%$ \\
\hline$h_{1}$ & 430 & 412 & $0.38 \%$ \\
\hline$v_{1}$ & 680 & 523 & $1.41 \%$ \\
\hline \hline$a_{2}$ & 1500 & 1452 & $2.42 \%$ \\
\hline$b_{2}$ & 0.5 & 0.36 & $2.25 \%$ \\
\hline$h_{2}$ & 400 & 398 & $0.67 \%$ \\
\hline$v_{2}$ & 680 & 651 & $0.58 \%$ \\
\hline \hline$a_{3}$ & 7500 & 7364 & $3.48 \%$ \\
\hline$b_{3}$ & 0.7 & 0.37 & $5.11 \%$ \\
\hline$h_{3}$ & 390 & 348 & $0.91 \%$ \\
\hline$v_{3}$ & 680 & 646 & $0.92 \%$ \\
\hline
\end{tabular}

After convergence, Tabs. 1, 2 and 3 report the estimated values for all the parameters and compares them to the ground truth values. Error rates for every parameter estimation are also provided. Each table contains the theoretical values as well as the estimated values for each region for comparing the obtained results. The considered ground truth values (from image of Fig. 3) of the hyperparameter vectors are $\theta=\{\mathbf{a}=[200,1500,7500], \mathbf{b}=[0.3,0.5,0.7], \mathbf{h}=$ $[430,400,390], \mathbf{v b}=[680,680,680]\}$. 
Table 2 Hyperboles parameters for $\sigma_{n}^{2}=15$ : estimated and reference values. Average error is $2.67 \%$ and minimal error is $1.06 \%$

\begin{tabular}{|c|c|c|c|}
\hline Parameters & Ground truth & Estimated value & Error rate \\
\hline$a_{1}$ & 200 & 173 & $0.39 \%$ \\
\hline$b_{1}$ & 0.3 & 0.19 & $1.05 \%$ \\
\hline$h_{1}$ & 430 & 424 & $0.11 \%$ \\
\hline$v_{1}$ & 680 & 1447 & $1.01 \%$ \\
\hline \hline$a_{2}$ & 1500 & 0.39 & $1.67 \%$ \\
\hline$b_{2}$ & 302 & $1.35 \%$ \\
\hline$h_{2}$ & 6.5 & 7474 & $1.21 \%$ \\
\hline$v_{2}$ & 400 & 0.52 & $0.10 \%$ \\
\hline \hline$a_{3}$ & 373 & $2.42 \%$ \\
\hline$b_{3}$ & 7500 & 662 & $3.08 \%$ \\
\hline$h_{3}$ & 0.7 & & $2.98 \%$ \\
\hline$v_{3}$ & 390 & & $0.31 \%$ \\
\hline
\end{tabular}

Table 3 Hyperboles parameters for $\sigma_{n}^{2}=5$ : estimated and reference values. Average error is $1.43 \%$ and minimal error is $0.87 \%$

\begin{tabular}{|c|c|c|c|}
\hline Parameters & Ground truth & Estimated value & Error rate \\
\hline$a_{1}$ & 200 & 189 & $0.11 \%$ \\
\hline$b_{1}$ & 0.3 & 0.24 & $0.66 \%$ \\
\hline$h_{1}$ & 430 & 526 & $0.09 \%$ \\
\hline$v_{1}$ & 680 & 1488 & $0.08 \%$ \\
\hline \hline$a_{2}$ & 0.38 & $0.83 \%$ \\
\hline$b_{2}$ & 376 & $1.07 \%$ \\
\hline$h_{2}$ & 1500 & 602 & $0.65 \%$ \\
\hline$v_{2}$ & 0.5 & 7489 & $1.17 \%$ \\
\hline \hline$a_{3}$ & 400 & 0.63 & $1.14 \%$ \\
\hline$b_{3}$ & 680 & 513 & $0.89 \%$ \\
\hline$h_{3}$ & 7500 & & $1.31 \%$ \\
\hline$v_{3}$ & 0.7 & 592 & $0.40 \%$ \\
\hline
\end{tabular}

We found that after the convergence of the algorithm, each time we reduce the variance value $\sigma_{n}^{2}$, the mean and minimum errors were also reduced. As shown in the tables, the different errors values started at around $3.64 \%$ as an average error and $2.31 \%$ for the minimal error for $\sigma_{n}^{2}=30$, and passed below $1.43 \%$ and $0.87 \%$ for the average and minimal error,respectively, for $\sigma_{n}^{2}=5$. The reported values clearly indicate the capacity of the proposed method to accurately estimate the parameters involved and thus to accurately characterize the different hyperbolas.

The most challenging part in road lane estimation is the case of sharp curvature usually located near the horizon line. All of parameters of the hyperbolic model are quite important but the parameter 'a' remains the most interesting and need to be carefully and precisely estimated. By approaching to the horizon line, the variation of parameter 'a' should be quite sensitive: a wrong estimation of parameter 'a' could causes a very wrong estimation of the 
curvature.

After running the Gibbs sampling algorithm several times, the difference results show the evaluation of the estimated parameters. The actual values of the hyperparameters of the vector (theta) are in confidence intervals of about $97 \%$. The different values obtained are very reliable and close to the initial results of the ground truth. Commonly, estimating the curvature parameters of lane marking in far regions is quite difficult to handle especially in the case of strong curvature. As shown in tables 1,2 and 3, we have found that the degree of confidence of the parameter "a" is very encouraging, with Error Rate values within the range of $1 \%$ and $3 \%$ for differents $\sigma_{n}^{2}$ which shows the accuracy of our estimators. The same performance for the other parameters "b", "h", and "v" which are very close to the theoretical values having an error rate less than $3 \%$.

\subsection{Real Data}

The lane marking detection algorithm described in this paper has been implemented on an Intel i7, 2.40 GHz CPU. The algorithm was evaluated on the ROMA dataset with available ground truth Veit et al. (2008). This database contains heterogeneous real road images acquired in very various conditions (full or partial shadow, different illumination conditions, etc.). The parameter $N$, which corresponds to the number of horizontal regions, is empirically set to 3 from the horizon line to the bottom of the image. In the bottom region, lines are typically straights, and the curvature is progressive from the second to the top region. Indeed, the choice of $N=3$ is argued by the progressive curvature level between the image top and bottom.

Beside the challenging real images of ROMA dataset, the availability of ground truth corresponding to road markings give us a best opportunity to assess lane detection and curvature estimation. The lane detection part was deeply evaluated in a previous work and by using the same dataset as used in that experiments Fakhfakh et al. (2018).

For different scenes and experimental conditions, Fig. 4 illustrates the performance of our method in lane characterization under different experimental conditions like high illumination, shadow, sharp curvature. Red lines in Fig 4 illustrate the output of our algorithm for lane and curvature estimation which overlay with the real road marking. The originality and novelty of our approach lie in the automatic estimation of all parameters of a hyperbolic model in each region of the road, unlike other works of the state of the art which generally estimates the degree of curvature by dividing the road regions into two parts. The reported results in this figure clearly show the originality and good performance of the proposed method for the automatic estimation of the different hyperbolic model parameters in each region and thus to accurately characterize the lanes. It is worth noting that these results have been obtained 
using the same iteration number and burn-in period as for simulated data, which correspond to a 10 seconds computational time on the used architecture.

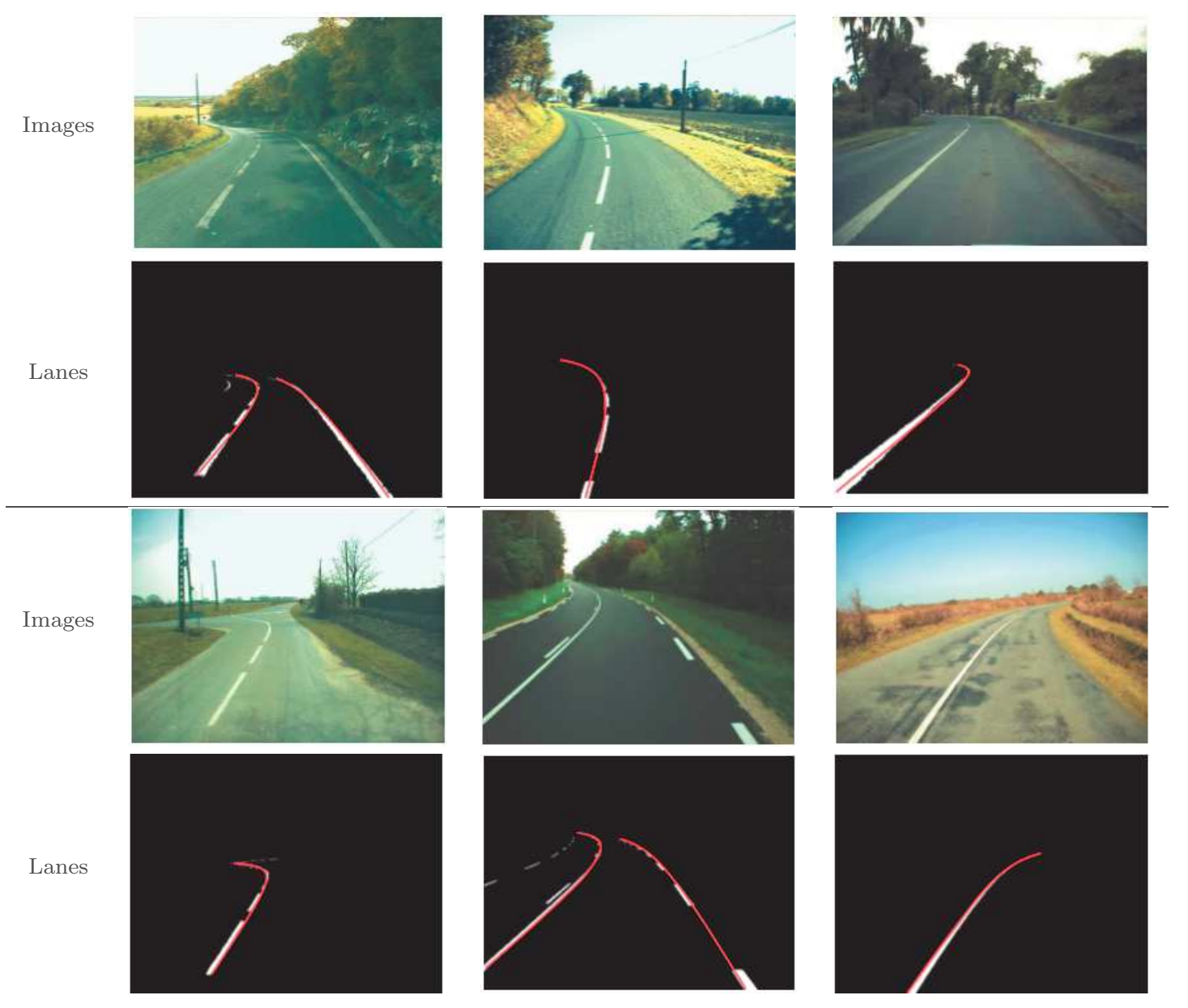

Fig. 4 Lane characterization using the proposed method.

In order to quantitatively evaluate the performance of our approach, the true positive rate $(\mathrm{TPR})$ and a false positive rate $(\mathrm{FPR})^{1}$ have been calculated to make comparisons with other state-of-the-art methods, specifically the works in Guo et al. (2015) and Tan et al. (2015) (detailed in Section 2). The reported values clearly show that the proposed method outperforms the other ones from a quantitative point of view. As shown in Table 4, the TPR rates are clearly better than other compared methods and lower for FPR.

$1 T P R=\frac{\# \text { detected lanes }}{\text { \#target lanes }}$ and $F P R=\frac{\# \text { false positive }}{\text { \# target lanes }}$. 
Table 4 TPR and FPR values for the proposed method and the works in Guo et al. (2015) and Tan et al. (2015).

\begin{tabular}{|c|c|c|c|c|c|c|}
\hline \multirow[t]{2}{*}{ Frames } & \multicolumn{2}{|c|}{ Method in Guo et al. (2015) } & \multicolumn{2}{|c|}{ Method in Tan et al. (2015) } & \multicolumn{2}{|c|}{ Our approach } \\
\hline & TPR & FPR & TPR & FPR & TPR & FPR \\
\hline 1 & $85.68 \%$ & $11.36 \%$ & $87.29 \%$ & $10.12 \%$ & $93.24 \%$ & $7.39 \%$ \\
\hline 2 & $93.91 \%$ & $4.02 \%$ & $94.41 \%$ & $3.88 \%$ & $95.20 \%$ & $4.52 \%$ \\
\hline 3 & $87.08 \%$ & $14.21 \%$ & $86.73 \%$ & $14.51 \%$ & $89.07 \%$ & $12.46 \%$ \\
\hline 4 & $83.18 \%$ & $29.98 \%$ & $85.04 \%$ & $21.03 \%$ & $88.67 \%$ & $15.37 \%$ \\
\hline 5 & $94.64 \%$ & $7.59 \%$ & $93.62 \%$ & $7.79 \%$ & $93.39 \%$ & $5.64 \%$ \\
\hline 6 & $89.54 \%$ & $10.74 \%$ & $90.33 \%$ & $9.34 \%$ & $91.45 \%$ & $7.89 \%$ \\
\hline Averages & $89.01 \%$ & $12.98 \%$ & $89.57 \%$ & $11.11 \%$ & $91.83 \%$ & $8.87 \%$ \\
\hline
\end{tabular}

The curvature estimation could be improved precisely for farthest regions because of the perspective geometry model of a camera, by either increasing the resolution of images or applying that approach in different image space representation, i.e. top view models.

\section{Conclusion}

In this study, we introduced a robust lane detection algorithm through curvature estimation. The proposed method is based on a mixture of hyperboles to model lanes. The image is decomposed horizontally, and the lane over each region is modeled using a hyperbola. The estimation task is formulated in a Bayesian framework, and the proposed method is fully automatic.

Reported results on both synthetic and real data demonstrate the good performance of the proposed method.

Future work will focus on using more efficient sampling scheme, such as Hamiltonian schemes, to accelerate the proposed MCMC procedure.

\section{References}

Aly M (2008) Real time detection of lane markers in urban streets. In: 2008 IEEE Intelligent Vehicles Symposium, IEEE, pp 7-12

Andrieu C (1998) Methodes mcmc pour l'analyse bayesienne de modeles de regression parametrique non lineaire. application a l'analyse de raies et a la deconvolution impulsionnelle. $\mathrm{PhD}$ thesis, Cergy-Pontoise

Antolovic D (2008) Review of the hough transform method, with an implementation of the fast hough variant for line detection. Department of Computer Science, Indiana University

Anwary AR, Yu H, Vassallo M (2018) Optimal foot location for placing wearable imu sensors and automatic feature extraction for gait analysis. IEEE Sensors Journal 18(6):2555-2567 
Arcos-García Á, Álvarez-García JA, Soria-Morillo LM (2018) Deep neural network for traffic sign recognition systems: An analysis of spatial transformers and stochastic optimisation methods. Neural Networks 99:158-165

Botekar NP, Mahalakshmi M (2017) Development of road sign recognition for adas using opencv. In: 2017 International Conference on Intelligent Computing and Control (I2C2), IEEE, pp 1-4

Bottazzi VS, Borges PV, Stantic B, Jo J (2014) Adaptive regions of interest based on hsv histograms for lane marks detection. In: Robot Intelligence Technology and Applications 2, Springer, pp 677-687

Bouguet JY (2001) Pyramidal implementation of the affine lucas kanade feature tracker description of the algorithm. Intel Corporation 5(1-10):4

Bresson G, Alsayed Z, Yu L, Glaser S (2017) Simultaneous localization and mapping: A survey of current trends in autonomous driving. IEEE Transactions on Intelligent Vehicles 2(3):194-220

Broggi A, Cerri P, Medici P, Porta PP, Ghisio G (2007) Real time road signs recognition. In: 2007 IEEE Intelligent Vehicles Symposium, IEEE, pp 981986

Cela AF, Bergasa LM, Sanchez FL, Herrera MA (2013) Lanes detection based on unsupervised and adaptive classifier. In: 2013 Fifth International Conference on Computational Intelligence, Communication Systems and Networks, IEEE, pp 228-233

Che G, Liu L, Yu Z (2019) An improved ant colony optimization algorithm based on particle swarm optimization algorithm for path planning of autonomous underwater vehicle. Journal of Ambient Intelligence and Humanized Computing pp 1-6

Chen Q, Wang H (2006) A real-time lane detection algorithm based on a hyperbola-pair model. In: 2006 IEEE Intelligent Vehicles Symposium, IEEE, pp 510-515

Chen Y, He M (2012) Sharp curve lane boundaries projective model and detection. In: IEEE 10th International Conference on Industrial Informatics, IEEE, pp 1188-1193

Chengping Y, Lincheng S, Dianle Z, Daibing Z, Zhiwei Z (2014) A new calibration method for vision system using differential gps. In: 2014 13th International Conference on Control Automation Robotics \& Vision (ICARCV), IEEE, pp 1514-1517

Chiu SH, Wen CY, Lee JH, Lin KH, Chen HM (2012) A fast randomized generalized hough transform for arbitrary shape detection. International Journal of Innovative Computing, Information and Control 8(2)

Cimurs R, Hwang J, Suh IH (2017) Bezier curve-based smoothing for path planner with curvature constraint. In: 2017 First IEEE International Conference on Robotic Computing (IRC), IEEE, pp 241-248

Cowles MK, Carlin BP (1996) Markov chain monte carlo convergence diagnostics: a comparative review. Journal of the American Statistical Association 91(434):883-904

Dagnino C, Lamberti P, Remogna S (2015) Curve network interpolation by c1 quadratic b-spline surfaces. Computer Aided Geometric Design 40:26-39 
Faizal ERM, Mansor HMAH (2009) Virtual mid-line detection on curve road for user guidance using simulation model. In: 2009 International Conference on Computer Technology and Development, IEEE, vol 1, pp 24-27

Fakhfakh M, Fakhfakh N, Chaari L (2018) Robust lane extraction using twodimension declivity. In: International Conference on Artificial Intelligence and Soft Computing, Springer, pp 14-24

Fan C, Xu Jb, Di S (2013) Lane detection based on machine learning algorithm. Telkomnika Indonesian Journal of Electrical Engineering 12(2):1403-1409

Guo J, Wei Z, Miao D (2015) Lane detection method based on improved ransac algorithm. In: 2015 IEEE Twelfth International Symposium on Autonomous Decentralized Systems, IEEE, pp 285-288

Guo SY, Kong YG, Tang Q, Zhang F (2008) Probabilistic hough transform for line detection utilizing surround suppression. In: 2008 International Conference on Machine Learning and Cybernetics, IEEE, vol 5, pp 2993-2998

Hassanein AS, Mohammad S, Sameer M, Ragab ME (2015) A survey on hough transform, theory, techniques and applications. arXiv preprint arXiv: 150202160

Ieng SS, Vrignon J, Gruyer D, Aubert D (2005) A new multi-lanes detection using multi-camera for robust vehicle location. In: IEEE Proceedings. Intelligent Vehicles Symposium,, IEEE, pp 700-705

Izadinia H, Sadeghi F, Ebadzadeh MM (2009) Fuzzy generalized hough transform invariant to rotation and scale in noisy environment. In: 2009 IEEE International Conference on Fuzzy Systems, IEEE, pp 153-158

Jang HJ, Baek SH, Park SY (2014) Curved lane detection using robust feature extraction. In: The 2014 2nd International Conference on Systems and Informatics (ICSAI 2014), IEEE, pp 109-112

Jung CR, Kelber CR (2005) Lane following and lane departure using a linearparabolic model. In: 2005 Image and Vision Computing, vol 23, pp $1192-$ 1202

Khalifa OO, Khan IM, Assidiq AA, Abdulla AH, Khan S (2010) A hyperbolapair based lane detection system for vehicle guidance. In: Proceedings of the World Congress on Engineering and Computer Science, vol 1, pp 978-988

Kim H (2019) Multiple vehicle tracking and classification system with a convolutional neural network. Journal of Ambient Intelligence and Humanized Computing pp 1-12, doi:10.1007/s12652-019-01429-5

Kim J, Lee M (2014) Robust lane detection based on convolutional neural network and random sample consensus. In: International Conference on Neural Information Processing, Springer, pp 454-461

Kim J, Park C (2017a) End-to-end ego lane estimation based on sequential transfer learning for self-driving cars. In: Proceedings of the IEEE Conference on Computer Vision and Pattern Recognition Workshops, pp 30-38

Kim J, Kim J, Jang GJ, Lee M (2017) Fast learning method for convolutional neural networks using extreme learning machine and its application to lane detection. Neural Networks 87:109-121

Kim T, Park T (2017b) Calibration method between dual 3d lidar sensors for autonomous vehicles. In: 2017 56th Annual Conference of the Society of 
Instrument and Control Engineers of Japan (SICE), IEEE, pp 1075-1081

Kultanen P, Xu L, Oja E (1990) Randomized hough transform (rht). In: [1990] Proceedings. 10th International Conference on Pattern Recognition, IEEE, vol 1, pp 631-635

Lee M, Han KY, Yu J, Lee YS (2019) A new lane following method based on deep learning for automated vehicles using surround view images. Journal of Ambient Intelligence and Humanized Computing pp 1-14, doi:10.1007/s12652-019-01496-8

Li J, Mei X, Prokhorov D, Tao D (2017a) Deep neural network for structural prediction and lane detection in traffic scene. IEEE transactions on neural networks and learning systems 28(3):690-703

Li M, Li Y, Jiang M (2018) Lane detection based on connection of various feature extraction methods. Advances in Multimedia

Li S, Xu J, Wei W, Qi H (2017b) Curve lane detection based on the binary particle swarm optimization. In: 2017 29th Chinese Control And Decision Conference (CCDC), IEEE, pp 75-80

Lim KH, Seng KP, Ang LM (2012) River flow lane detection and kalman filtering-based b-spline lane tracking. International Journal of Vehicular Technology 2012

Lin Q, Han Y, Hahn H (2010) Real-time lane departure detection based on extended edge-linking algorithm. In: 2010 Second International Conference on Computer Research and Development, IEEE, pp 725-730

Liu J, Lou L, Huang D, Zheng Y, Xia W (2018) Lane detection based on straight line model and k-means clustering. In: 2018 IEEE 7th Data Driven Control and Learning Systems Conference (DDCLS), IEEE, pp 527-532

Liu X, Wang G, Liao J, Li B, He Q, Meng MQH (2012) Detection of geometric shape for traffic lane and mark. In: 2012 IEEE International Conference on Information and Automation, IEEE, pp 395-399

Lopez-Krahe J, Pousset P (1988) Transformée de hough discrete et bornée, applicationa la detection de droites paralleles et du réseau routier. Traitement du signal 5(4):281-290

Merali RS, Barfoot TD (2013) Occupancy grid mapping with markov chain monte carlo gibbs sampling. In: 2013 IEEE International Conference on Robotics and Automation, IEEE, pp 3183-3189

Mukhopadhyay P, Chaudhuri BB (2015) A survey of hough transform. Pattern Recognition 48(3):993-1010

Nguyen TT, Spehr J, Lin THH, Lipinski D (2015) Fused raised pavement marker detection using 2d-lidar and mono camera. In: IEEE 18th International Conference on Intelligent Transportation Systems, IEEE, pp 23462351

Parajuli A, Celenk M, Riley HB (2013) Robust lane detection in shadows and low illumination conditions using local gradient features. Open Journal of Applied Sciences 3(01):68

Roumeliotis SI, Bekey GA (2000) Bayesian estimation and kalman filtering: A unified framework for mobile robot localization. In: Proceedings 2000 ICRA. Millennium Conference. IEEE International Conference on Robotics 
and Automation. Symposia Proceedings (Cat. No. 00CH37065), IEEE, vol 3, pp 2985-2992

Santana DDS, Furukawa CM, Maruyama N (2013) Sensor fusion with lowgrade inertial sensors and odometer to estimate geodetic coordinates in environments without gps signal. IEEE Latin America Transactions 11(4):1015 1021

Saxena P, Gupta N, Laskar SY, Borah PP (2015) A study on automatic detection and recognition techniques for road signs. Int J Comput Eng Res $5(12): 24-28$

Shenoy MV, Karuppiah A, Manjarekar N (2019) A lightweight ann based robust localization technique for rapid deployment of autonomous systems. Journal of Ambient Intelligence and Humanized Computing pp 1-16, doi:10.1007/s12652-019-01331-0

Shiffrin RM, Lee MD, Kim W, Wagenmakers EJ (2008) A survey of model evaluation approaches with a tutorial on hierarchical bayesian methods. Cognitive Science 32(8):1248-1284

Song W, Yang Y, Fu M, Li Y, Wang M (2018) Lane detection and classification for forward collision warning system based on stereo vision. IEEE Sensors Journal 18(12):5151-5163

Tan H, Zhou Y, Zhu Y, Yao D, Li K (2014) A novel curve lane detection based on improved river flow and ransa. In: 17th International IEEE Conference on Intelligent Transportation Systems (ITSC), IEEE, pp 133-138

Tan H, Zhou Y, Zhu Y, Yao D, Wang J (2015) Improved river flow and random sample consensus for curve lane detection. Advances in Mechanical Engineering 7(7):1687814015593866

Thomas AD (1992) Compressing the parameter space of the generalised hough transform. Pattern Recognition Letters 13(2):107-112

Timar Y, Alagoz F (2010) Lane detection for intelligent vehicles in challenging scenarios. In: 2010 2nd International Conference on Computational Intelligence, Communication Systems and Networks, IEEE, pp 37-43

Veit T, Tarel JP, Nicolle P, Charbonnier P (2008) Evaluation of road marking feature extraction. In: 2008 11th International IEEE Conference on Intelligent Transportation Systems, IEEE, pp 174-181

Wang J, An X (2010) A multi-step curved lane detection algorithm based on hyperbola-pair model. In: 2010 IEEE International Conference on Automation and Logistics, IEEE, pp 132-137

Wang Y, Teoh EK, Shen D (1999) Lane detection using b-snake. In: Proceedings 1999 International Conference on Information Intelligence and Systems (Cat. No. PR00446), IEEE, pp 438-443

Wang Y, Bai L, Fairhurst M (2008) Robust road modeling and tracking using condensation. IEEE Transactions on Intelligent Transportation Systems $9(4): 570$

Wu Y, Chen Z (2016) A detection method of road traffic sign based on inverse perspective transform. In: 2016 IEEE International Conference of Online Analysis and Computing Science (ICOACS), IEEE, pp 293-296 
Xu L, Oja E, Kultanen P (1990) A new curve detection method: randomized hough transform (rht). Pattern recognition letters 11(5):331-338

Zhou Y, Dong Z (2017) A vision-based autonomous detection scheme for obstacles on the runway. In: 2017 Chinese Automation Congress (CAC), IEEE, pp $832-838$ 